\title{
Comparação entre sistemas para criação de acervos digitais: análise dos softwares livres DSpace, EPrints, Fedora, Greenstone e Islandora a partir de novas dimensões analíticas
}

\author{
Comparison between systems for creation of digital collections: analysis of free software \\ DSpace, EPrints, Fedora, Greenstone and Islandora from new analytical dimensions
}

\begin{abstract}
Dalton Lopes Martins
Doutor em Ciência da Informação pela Escola de Comunicação e Artes da Universidade de São Paulo -

ECA/USP.

Professor no curso de Biblioteconomia da Faculdade de Ciência da Informação na Universidade de Brasília UnB e no Programa de Pós-graduação em Comunicação da Faculdade de Informação e Comunicação da Universidade Federal de Goiás - UFG.

E-mail: dmartins@gmail.com Marcel Ferrante Silva
Doutor em Ciência da Informação pela Universidade Federal de Minas Gerais - UFMG.
Professor Adjunto no Curso de Gestão da Informação da Faculdade de Informação e Comunicação da
Universidade Federal de Goiás - UFG.
E-mail: marcelf@ gmail.com

Joyce Siqueira Mestre em Ciência da Computação pela Universidade Federal de Goiás (UFG). Professora da Faculdade Anhanguera de Anápolis, Goiás, Brasil. E-mail: joycitta@gmail.com
\end{abstract}

\section{Resumo}

Os repositórios digitais estão cada vez mais integrados ao cotidiano, principalmente por possibilitar a universalização do conhecimento por meio de acesso rápido e, muitas vezes, gratuito a documentos. Existem atualmente diversas opções de softwares disponíveis para este fim, dessa forma, para auxiliar os gestores responsáveis por definir a melhor ferramenta para seu projeto, originou-se o objetivo desta pesquisa, de analisar cinco principais softwares livres: DSpace, EPrints, Fedora, Greenstone e Islandora e verificar quais destes atendem o maior número de requisitos previamente classificados em dimensões analíticas. Assim, foram analisadas 182 funcionalidades, divididas em 10 dimensões analíticas, dos quais obteve-se que o DSpace atende uma maior porcentagem de funcionalidades, com 57\%, sendo seguido pelo EPrints, com 38\%, a Islandora, com 36\%, o Greenstone, com $23 \%$ e o Fedora, com 19\%. A partir deste resultado, pretende-se oferecer aos gestores importante ferramenta de decisão.

Palavras-chave: Software livre. Repositórios digitais. Dimensões analíticas.

\begin{abstract}
Digital repositories are increasingly integrated into daily life, mainly because it makes possible the universalization of knowledge through quick and often free access to documents. There are currently several software options available for this purpose, so to help managers responsible for defining the best tool for their project, the goal of this research was to analyze five main open source software: DSpace, EPrints, Fedora, Greenstone and Islandora and verify which of them meet the highest number of requirements previously classified in analytical dimensions. Thus, we analyzed 182 functionalities, divided into 10 analytical dimensions, from which it was obtained that DSpace meets a greater percentage of functionalities, with $57 \%$, followed by EPrints, with $38 \%$, Islandora, with $36 \%$, Greenstone, with $23 \%$ and Fedora, with $19 \%$. From this result, it is intended to offer managers an important decision tool.
\end{abstract}

Keywords: Open source software. Digital repositories. Analytical dimensions 


\section{Introdução}

A criação e desenvolvimento de acervos digitais no Brasil têm chamado a atenção pelas dificuldades e desafios, mas também pelas oportunidades e benefícios que podem trazer. Em anos recentes, o Ministério da Cultura deu passos importantes e assumiu uma posição protagonista para a área, ao realizar, no ano de 2010, o Simpósio Internacional para Políticas Públicas para Acervos Digitais (MINISTÉRIO DA CULTURA, 2010) e, em 2015, propor a criação de uma Política Nacional de Acervos Digitais (MINISTÉRIO DA CULTURA, 2015).

Essas ações visavam atender a meta 40, do Plano Nacional de Cultura, que prevê que, até 2020, estejam disponíveis na internet: as obras audiovisuais da Cinemateca Brasileira e do Centro Técnico Audiovisual (CTAv); o acervo da Fundação Casa de Rui Barbosa; os inventários e ações de reconhecimento realizados pelo Instituto do Patrimônio Histórico e Artístico Nacional (Iphan); as obras de autores brasileiros do acervo da Fundação Biblioteca Nacional (FBN) e o acervo iconográfico, sonoro e audiovisual do Centro de Documentação da Fundação Nacional das Artes (Cedoc/Funarte). Além disso, 100\% das bibliotecas públicas e $70 \%$ dos museus e arquivos deverão disponibilizar informações sobre seus acervos no Sistema Nacional de Informações e Indicadores Culturais (MINISTÉRIO DA CULTURA, 2011).

Como se observa, os desafios colocados são ousados e de difícil realização no contexto econômico atual, no entanto, sinalizam parâmetros que podem auxiliar na discussão científica da área da Ciência da Informação, na busca por soluções que não apenas auxiliem no atendimento da meta, mas também na construção de perspectivas de interoperabilidade entre diferentes acervos, permitindo que o processo de digitalização da cultura brasileira possa ser acessado e socializado de maneira simples, pela população do país.

Tendo isso em vista e considerando que soluções de tamanha escala necessitarão de baixo custo para poderem se espalhar pelo país e ainda que o software no qual esses acervos serão disponibilizados é fundamental para que as soluções possam ser escaláveis, realiza-se, na presente pesquisa, a comparação entre os principais softwares livres, segundo a literatura da área, voltados para a construção de acervos digitais. Entende-se por software livre aquele que autoriza expressamente em sua licença de uso, qualquer usuário copiar, distribuir e usar, com ou sem modificações, gratuitamente.

O objetivo é evidenciar as vantagens, potencialidades e fragilidades dos sistemas disponíveis, de maneira a facilitar a tomada de decisão por gestores e pesquisadores da área 
quando da necessidade de escolha de um software livre para seus acervos. Além disso, procurase encontrar áreas críticas, sobretudo quando se consideram novas possibilidades de colaboração em rede em torno de objetos digitais e a interoperabilidade com as mídias sociais, características atualmente presentes nos principais ambientes disponíveis na Internet no que hoje chamamos de Web Social.

Para alcançar os objetivos supracitados, os softwares foram avaliados e, sobretudo, comparados de maneira a levar em consideração seus recursos técnicos disponíveis, classificados em diferentes dimensões analíticas. Dessa forma, o artigo está organizado a partir da seção de Revisão Bibliográfica, onde se apresenta pesquisas de referência sobre os desafios relacionados a softwares para acervos digitais e as principais opções de código livre para este fim. A partir da identificação destes softwares, apresenta-se na subseção Softwares Livres para Acervos Digitais cada um deles, assim como suas principais características. Na sequência, apresentam-se os Procedimentos Metodológicos adotados para realização da comparação entre os softwares, e ainda, partindo dos indicadores propostos por Martins e Silva (2017), que apresenta critérios de comparação e dimensões analíticas pelas quais eles são organizados. Na seção Resultados apresentam-se as potencialidades e lacunas identificadas. Por fim, discutem-se, na seção Conclusão, alguns desdobramentos, evidências e critérios que possam auxiliar na tomada de decisão por gestores.

\section{Revisão bibliográfica}

As pesquisas sobre comparação entre softwares para a criação de acervos digitais, e mesmo bibliotecas digitais, são ainda muito restritas no Brasil. Em uma busca realizada em março de 2017, na Base de Dados em Ciência da Informação/BRAPCI, usando os termos de busca software+"bibliotecas digitais" e software+"acervos digitais" foram encontrados apenas 8 artigos na primeira busca e 2 na segunda, sendo que nenhum destes apresenta pesquisa de comparação entre softwares, mas sim, relatos sobre casos de aplicação de um software específico (PIZZORNO, 2005); apresentação de questões de funcionalidades, como segurança da informação para preservação digital (SUNYE, 2010) ou questões relacionadas a padrões universais, como o caso da web semântica para publicação de informação na web (AMBINDER; MARCONDES, 2013).

Cientes da necessidade de expandir o campo de busca para localizar publicações que 
apontassem os softwares livres que poderiam ser analisados, assim como os critérios que poderiam ser utilizados para compará-los, utilizou-se o trabalho de Martins e Silva (2017), que consultou duas bases de dados de representatividade internacional, a Web of Science e o Google Acadêmico. Lá foram identificados 07 relevantes estudos comparativos, utilizados como prévia para a proposta de um novo conjunto de critérios de comparação e organização, classificados em dimensões analíticas. Os trabalhamos utilizados como referência são Bankler e Gleason (2014), Patel e outros (2014), Kumar e Raghunadha (2013), Randhawa (2012), Tramboo e outros (2012), Lihitkar e Lihitkar (2012), Goh e outros (2006).

As dimensões analíticas são utilizadas neste trabalho como indicador de comparação entre os softwares analisados. São consideradas 10 dimensões, descritas brevemente a seguir, com 182 funcionalidades: Sistema de Busca (11) permite encontrar algo por meio da simples pesquisa por palavra-chave ou busca avançada; Sistema de Navegação (11) provê maneiras para o usuário navegar, filtrar e encontrar uma informação; Sistema de Organização (12) trata sobre como o conteúdo é categorizado; Sistema de Rotulagem e Indexação (07) envolve a descrição ou representação de conteúdo; Sistema de Preservação Digital (18) traz questões relacionadas à preservação de longo prazo das informações, tais como teste de integridade, versionamento, migração do formato, metadados de preservação; Sistema de Interoperabilidade (20) agrupa requisitos sobre as formas de intercâmbio de informações do repositório digital com outros sistemas de informação, para importação e exportação por meio de vários protocolos e diferentes formatos; Sistema de Administração (38) classifica os requisitos referentes ao controle dos usuários, grupos e permissões; Sistema de Colaboração e Interação Social (36) inclui requisitos que estimulam o engajamento da comunidade de usuários na produção, descrição e organização do conteúdo, propondo, de certa maneira, novas possibilidades de explorar formas de organização de processo informacional, ativando inteligências e incluindo a possibilidade de usuários atuarem em etapas que tradicionalmente estão destinadas apenas para profissionais da informação; Sistema de Suporte e Manutenção (07), reúne requisitos externos ao sistema de informação, mas considerados de grande importância para adoção de um repositório, tais como, o suporte e manutenção, documentação, listas de e-mail, gestão de falhas; Características Gerais (22) no qual foram agrupados os requisitos que são intrínsecos ao ambiente do sistema de informação tais como: arquitetura de servidores, banco de dados, linguagens de programação, design, licença do software, visualizadores, línguas, atualização, geolocalização e cache. As dimensões, assim como suas funcionalidades foram apresentadas em Martins e Silva (2017). 
Também a partir desta revisão bibliográfica que se chegou a menção dos principais softwares livres estudados por pesquisadores da área para a gestão de acervos digitais, sendo eles: DSpace, EPrints, Fedora, Greenstone e Islandora, que são apresentados na próxima seção.

\section{Softwares livres para acervos digitais}

Os softwares livres são apresentados a partir do histórico de sua concepção descrito em seus respectivos sites. Além disso, descreve-se de maneira sucinta o objetivo das ferramentas e algumas de suas principais características base.

\section{DSpace $^{1}$}

O DSpace encontra-se, em 2018, na versão 6.x e foi concebido do esforço colaborativo entre a MIT Libraries e a Hewlett-Packard Company com o propósito de possibilitar a criação de repositórios digitais com funções de armazenamento, gerenciamento, preservação e visibilidade da produção intelectual de forma que possa ser facilmente adotado e adaptado por instituições de diversos segmentos. Possui uma arquitetura que permite o gerenciamento da produção científica em qualquer tipo de material digital, como arquivos de áudio e vídeo, páginas web, coleções de bibliotecas digitais, livros, teses, programas de computador, entre outros. Possui natureza operacional específica de preservação de objetos digitais, sendo esta iniciativa de grande interesse da comunidade científica.

\footnotetext{
${ }^{1}$ http://dspace.org/introducing
} 


\section{EPrints $^{2}$} analíticas

O EPrints encontra-se, em 2018, na versão v3.3.15 (2018) e permite aos seus usuários a construção de repositórios de acesso aberto compatíveis com o Open Archives Initiative Protocol for Metadata Harvesting. Compartilha muitas das características comumente observadas em sistemas de gestão de documentos, mas é usado principalmente para repositórios institucionais e periódicos científicos. Foi desenvolvido pela School of Electronics and Computer Science na University of Southampton e foi disponibilizado sob a licença GPL, General Public License. Foi concebido com objetivo de criar um repositório web altamente configurável, sendo geralmente aplicado não somente como um repositório aberto para pesquisa de artigos, mas também para outros tipos de arquivo como imagens, dados, áudio, ou qualquer item que possa ser armazenado digitalmente.

\section{Fedora $^{3}$}

Fedora encontra-se, em 2018, na versão 28 e é um software robusto e modular para a gestão e difusão de conteúdos digitais. Foi originalmente desenvolvido por pesquisadores da Cornell University com uma arquitetura inspirada no framework Kahn and Wilensky que implementa uma camada de funcionalidades voltadas para o armazenamento, gerenciamento e acesso aos conteúdos na forma de objetos digitais. Define um conjunto de abstrações para expressar objetos digitais, estabelecendo relações entre eles, e ligando "comportamentos" (isto é, serviços) para objetos digitais. É especialmente adequado para bibliotecas e arquivos digitais, tanto para o acesso como para preservação. Também é usado para fornecer acesso especializado para coleções digitais muito grandes e complexas, como de materiais históricos e culturais, bem como dados científicos. O Fedora tem uma comunidade de usuários disseminada por todo o mundo que inclui desde organizações de herança cultural e acadêmica, universidades e instituições de pesquisa, bibliotecas nacionais e agências governamentais.

\footnotetext{
${ }^{2}$ http://www.eprints.org/

${ }^{3}$ http://fedorarepository.org/about
} 


\section{Greenstone $^{4}$}

O Greenstone encontra-se, em 2018, na versão 3.08 e é voltado para a construção e distribuição de bibliotecas digitais. É desenvolvido e distribuído em cooperação com a UNESCO e as ONG Human Info por meio do New Zealand Digital Library Project na University of Waikato. Possui como objetivo a capacitação de usuários, especialmente em universidades, bibliotecas e outras instituições de serviço público, para a construção de suas próprias bibliotecas digitais. As bibliotecas digitais trazem na sua essência um mecanismo de organização e divulgação que reformula o modo como a informação é disseminada e adquirida em comunidades e instituições parceiras da UNESCO nos campos da educação, ciência e cultura em todo o mundo, e particularmente nos países em desenvolvimento.

\section{Islandora $^{5}$}

O Islandora encontra-se, em 2018, na versão 7.x-1.10 e foi desenvolvido com o objetivo de ajudar instituições e organizações a realizar o gerenciamento e descoberta colaborativa de seus ativos digitais (arquivos de áudio e vídeo, ebooks, etc) utilizando as melhores práticas recomendadas. Islandora foi originalmente desenvolvido pela biblioteca Robertson da University of Prince Edward Island's, tendo sua implementação e manutenção sob a responsabilidade de uma crescente comunidade internacional. Sua arquitetura foi construída em uma base formada por outros softwares, como o Drupal, Fedora, e Solr, onde o Islandora disponibiliza um conjunto de pacotes de soluções que permite aos seus usuários manipularem diversos tipos de objetos digitais, tais como imagens, vídeos, arquivos pdf, entre outros, e domínios de conhecimentos. Esses pacotes de soluções permitem a integração com os outros softwares, editores e aplicativos de processamento de dados e esquemas de metadados.

Uma vez apresentados os softwares que serão considerados para comparação, veremos a seguir os procedimentos metodológicos utilizados para a análise dos mesmos.

\footnotetext{
${ }^{4}$ http://www.greenstone.org/

${ }^{5}$ http://islandora.ca/about
} 


\section{Procedimentos metodológicos}

Os softwares são comparados a fim de averiguar seu grau de completude, facilitando a identificação dos que possuem mais ou menos funcionalidades, a partir de conjuntos específicos intitulados Dimensões Analíticas (MARTINS e SILVA, 2017). A Tabela 01 exibe as dimensões e a distribuição da quantidade de funcionalidades.

Tabela 01 - Distribuição de funcionalidades dos softwares, por dimensão analítica

\section{Dimensão Analítica}

Quantidade de

\begin{tabular}{lc}
\hline Sistema de Busca & 11 \\
\hline Sistema de Navegação & 11 \\
\hline Sistema de Organização & 12 \\
\hline Sistema de Rotulagem e Indexação & 07 \\
\hline Sistema de Preservação Digital & 18 \\
\hline Sistema de Interoperabilidade & 20 \\
\hline Sistema de Administração & 38 \\
\hline Sistema de Colaboração e Interação Social & 36 \\
\hline Sistema de Suporte e Manutenção & 07 \\
\hline Características Gerais & 22 \\
\hline TOTAL & $\mathbf{1 8 2}$
\end{tabular}

Fonte: dos autores

Considerando o contexto acima apresentado, procede-se aos seguintes passos técnicos para execução da pesquisa:

1. Instalação dos softwares livres em máquina virtual Linux Ubuntu Desktop, versão 16.04, LTS. Foram instaladas as seguintes versões dos softwares: EPrints 3.3.15, Fedora 19, Greenstone 3.08, DSpace 5.x e Islandora 7-x 1.5.

2. A instalação básica dos softwares contempla poucas funcionalidades, por isso, a análise é realizada a partir da interface, mas principalmente da documentação da versão atual de cada software e de outras fontes de pesquisa, tais como, website e fórum de usuários, quando disponíveis.

3. Criação de uma planilha eletrônica online, disponível no Google Drive, preenchida com a avaliação dos softwares. Para cada funcionalidade foi atribuído 1, quando existente e 0, quando inexistente. A planilha está disponível no link: https://goo.gl/kPzjvB, para qual utilizamos o encurtador de URL do Google.

a. Para cada funcionalidade para qual foi atribuído o valor 1 há comprovação por meio de print da tela. As figuras também estão disponíveis no link: https://goo.g1/kPzjvB. 
4. Sistematização das pontuações por meio de gráficos para comparação e avaliação dos resultados.

A seguir, apresentam-se os resultados obtidos.

\section{Resultados}

Os resultados são apresentados por dimensão analítica por meio de gráficos que sintetizam a quantidade de funcionalidades existentes em cada software. Entende-se que os que obtiverem mais pontos são os mais completos na dimensão específica. Além disso, destaca-se que os números finais são indicadores de completude das dimensões, pois a vasta documentação, nem sempre agrupada em um único local, dificulta a apresentação de números exatos.

\section{Sistema de busca}

Para o Sistema de Busca foram elencadas 11 funcionalidades, cujos resultados estão dispostos na Figura 01.

O DSpace atendeu todos os requisitos, seguido pelo EPrints, com 64\%, que, de maneira geral, atende a dimensão, apresentando pequenos déficits de opções para o usuário, tais como, busca com máscara ou por proximidade de expressão. O Greenstone, com 45\%, possui praticamente todos os itens para busca, no entanto, oferece poucas opções para ordenação dos resultados. O Islandora, com apenas $18 \%$, foca em uma busca simples, sem opções para busca avançada ou ordenação dos resultados. O Fedora, com $0 \%$, pode ser justificado por sua função principal, que é a de servir como back-end (ou o "lado do servidor", se refere a tudo o que o usuário não pode ver no navegador, como bancos de dados e servidores, por exemplo) para outras aplicações, que seriam então responsáveis por importantes interfaces. 
Figura 01 - Funcionalidades do sistema de busca.

Sistema de Busca

20

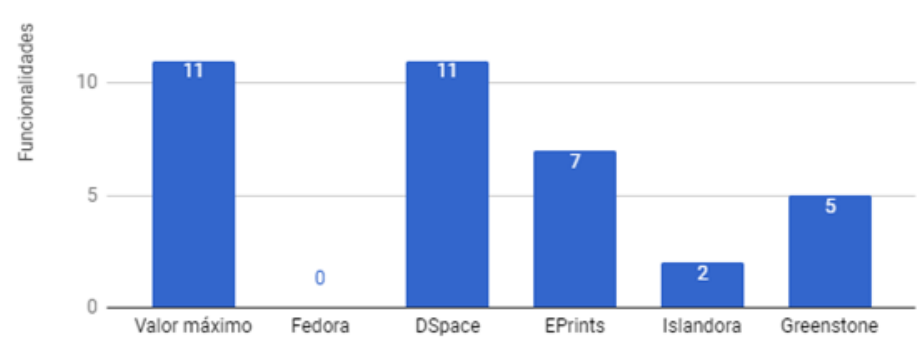

Fonte: dos autores.

Este sistema reflete diretamente na experiência do usuário, que tem como uma das finalidades principais, localizar arquivos de seu interesse, assim, dá-se destaque a completude do DSpace e também a quantidade de funcionalidades que podem ser futuramente implementadas nos outros repositórios.

\section{Sistema de navegação}

A Figura 02 demonstra os resultados obtidos no Sistema de Navegação.

Figura 02 - Funcionalidades do Sistema de Navegação

Sistema de Navegação

20

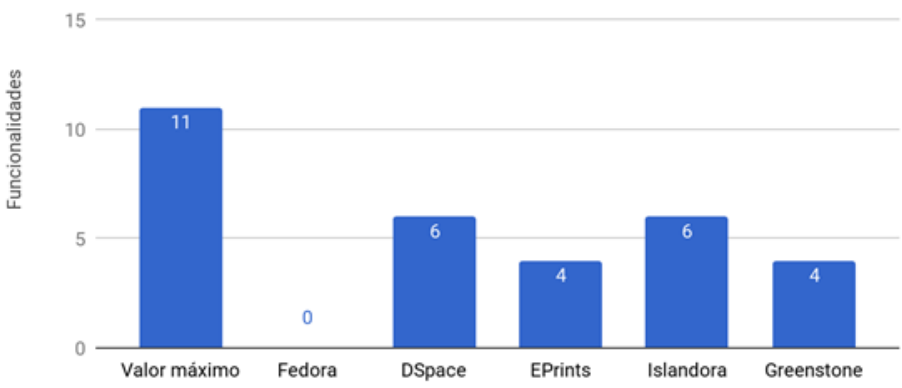

Fonte: dos autores.

Esta dimensão possui 11 itens, dos quais, o DSpace e o Islandora contemplam um maior número de funcionalidades, alcançando $55 \%$ de quesitos presentes. Logo abaixo, estão os repositórios EPrints e Greenstone, com 36\%. O Fedora também obteve 0\% neste quesito. 


\section{Sistema de organização}

A Figura 03 apresenta os resultados do Sistema de Organização.

Figura 03 - Funcionalidades do Sistema de Organização.

Sistema de Organização

20

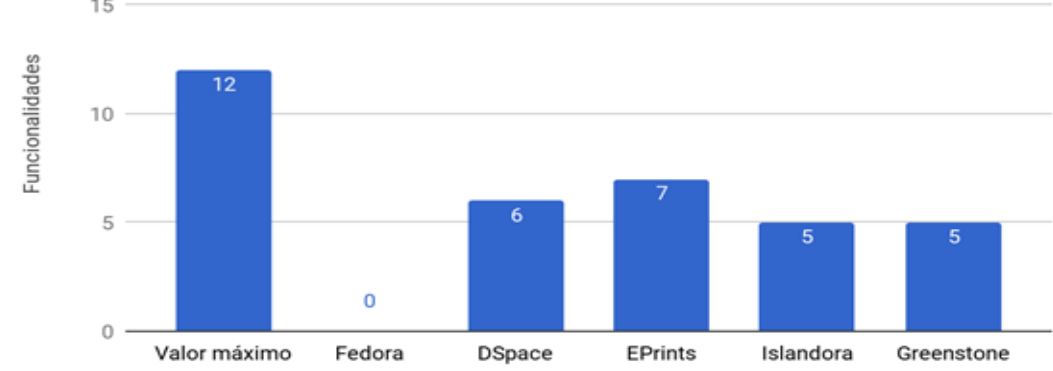

Fonte: dos autores.

Esta dimensão possui 12 funcionalidades, nas quais o repositório EPrints contempla um maior número de itens, alcançando 58\%. Empatados em segundo lugar, estão DSpace e o Islandora e Greenstone, com 50\% das funcionalidades. Na sequência, o Fedora, com 0\%. Para ilustrar, cita-se alguns requisitos não contemplados por nenhum dos repositórios: ordenação de resultados por votações e organização por taxonomia, tesauros e ontologias.

\section{Sistema de rotulagem e indexação}

O Sistema de Rotulagem e Indexação possui 7 funcionalidades, e os resultados da análise estão dispostos na Figura 04. 
Figura 04 - Funcionalidades do Sistema de Rotulagem e Indexação

Sistema de Rotulagem e Indexação

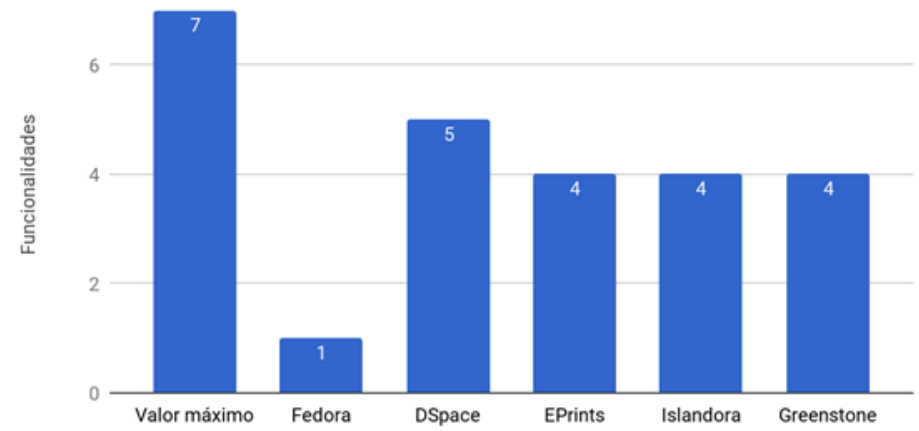

Fonte: dos autores

Nesta dimensão analítica o DSpace apresentou resultados superiores aos demais, contemplando $71 \%$ de itens. Em segundo lugar estão empatados os repositórios EPrints e Greenstone e Islandora, com 57\%. Por último, o Fedora, com 14\%. De forma geral, os itens menos presentes foram: Drag and Drop; tipos de campos do formulário de submissão e configuração de valores padrão para campos de metadados.

\section{Sistema de preservação digital}

Os resultados referentes ao Sistema de Preservação Digital estão apresentados na Figura 05.

Figura 05 - Funcionalidades do Sistema de Preservação Digital.

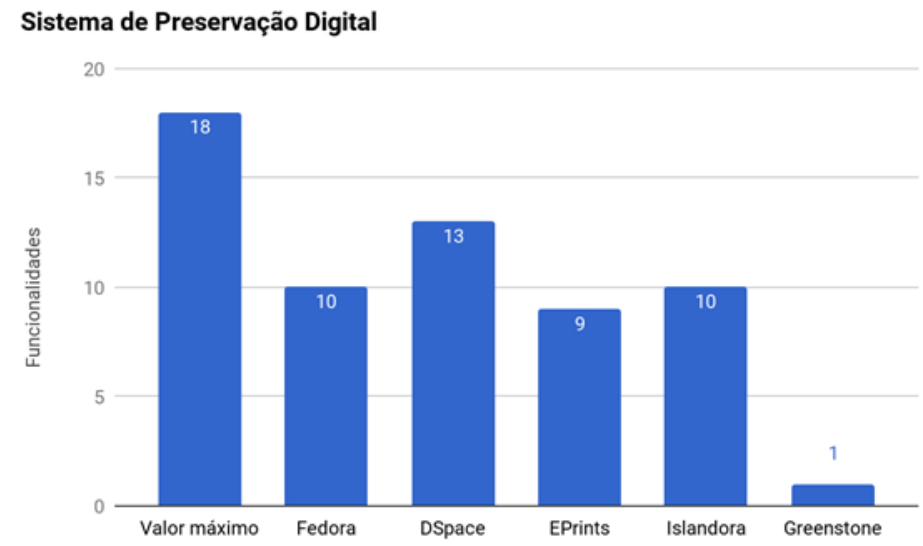

Fonte: dos autores

Nesta dimensão o DSpace, mais uma vez, se mostrou superior em relação aos demais, 
apresentando $72 \%$ de funcionalidades presentes, seguidos pelo Fedora e Islandora, ambos com $56 \%$, do EPrints, com $50 \%$ e o Greenstone com $6 \%$.

Neste item observa-se valores mais competitivos no Fedora, pois o Sistema de Preservação Digital está mais relacionado ao comportamento do repositório, do que a interface em si. Por outro lado, o Greenstone mostra-se claramente pouco desenvolvido.

\section{Sistema de interoperabilidade}

O Sistema de Interoperabilidade se destaca por ser um dos principais desafios que pesquisas na área apontam em relação aos softwares atuais, visto que permitiria que diferentes acervos se beneficiassem do potencial da web de conectar dados e informações de diferentes tipos, origens e representadas a partir de diferentes padrões de metadados. Os resultados da análise desta dimensão estão apresentados na Figura 06.

Figura 06 - Funcionalidades do Sistema de Interoperabilidade.

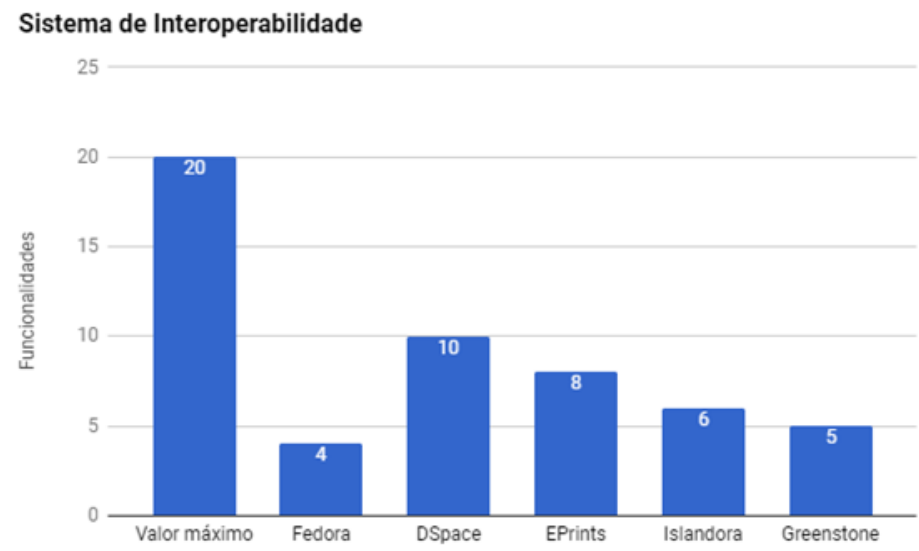

Fonte: dos autores

Este Sistema apresenta 20 funcionalidades. Como resultado obteve-se que o DSpace se mostra superior em relação aos demais, alcançando a marca de $50 \%$ de itens identificados. $\mathrm{Na}$ sequência, EPrints (40\%), Islandora (30\%), Greenstone (25\%) e Fedora (20\%).

No entanto, nota-se uma deficiência geral em relação a interação com as redes sociais, requisito para o qual foi atribuído valor 0 para todos os repositórios: importar vídeos do YouTube via URL, importar fotos automaticamente do Flirck ou do Facebook. Além disso, também não foram encontrados os protocolos de busca Dienst e SDLIP. 


\section{Sistema de administração}

Dimensão com maior número de funcionalidades, totalizando 38 requisitos. A análise do Sistema de Administração está demonstrada na Figura 07.

\section{Figura 07 - Funcionalidades do Sistema de Administração}

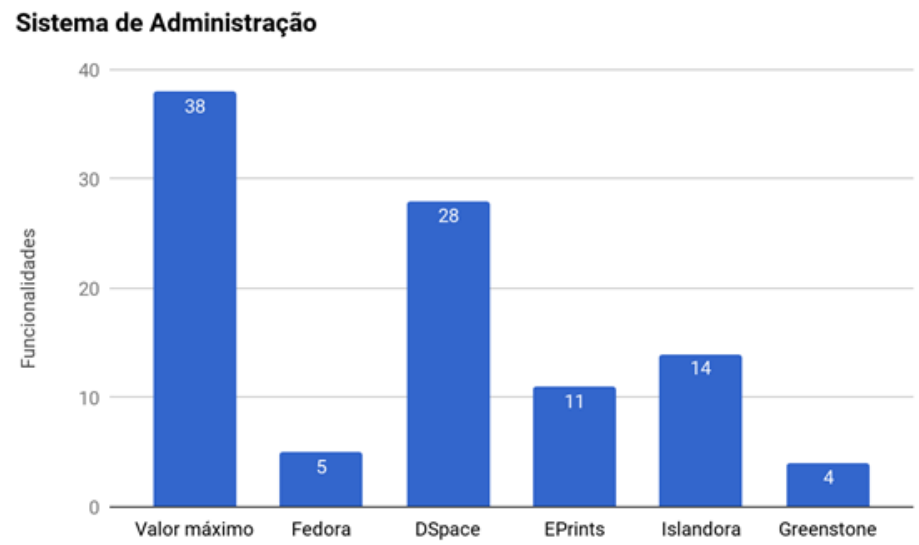

Fonte: dos autores.

O DSpace desponta como o repositório mais completo, com $74 \%$ das funcionalidades encontradas. Este resultado aponta uma grande diferença no número de funcionalidades entre o primeiro e os demais repositórios. Nas próximas colocações estão o Islandora, com 37\%, o EPrints, com $29 \%$, e o Fedora, com $13 \%$ e o Greenstone, com $11 \%$.

\section{Sistema de colaboração e interação social}

Na dimensão Sistema de Colaboração e Interação Social encontra-se a maior carência no atendimento dos requisitos, cujos resultados estão apresentados na Figura 08. 
Figura 08 - Funcionalidades do Sistema de Colaboração e Interação Social

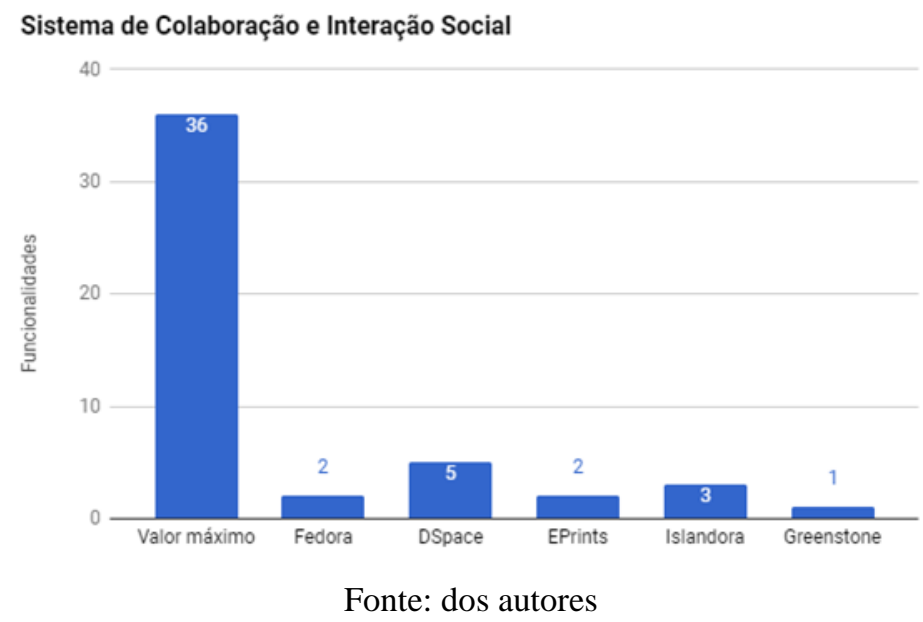

Este Sistema possui 36 funcionalidades. Em primeiro lugar, o DSpace possui apenas $14 \%$, seguido dos Islandora, com $8 \%$, o Fedora e o EPrints, com $6 \%$ e por último, o Greenstone (3\%), ou seja, os repositórios praticamente não oferecem recursos de colaboração ou interação social.

\section{Sistema de suporte e manutenção}

Esta dimensão obteve os melhores resultados, que são apresentados na Figura 09.

Figura 09 - Funcionalidades do Sistema de Suporte e Manutenção.

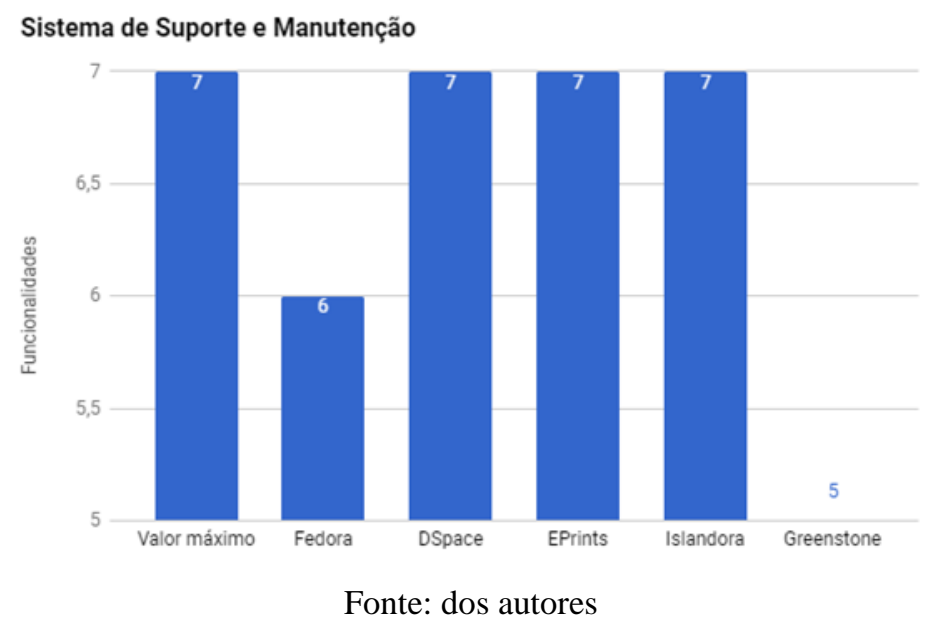

Diferente da dimensão analítica anterior, na dimensão Sistema de Suporte e Manutenção todos os repositórios estão bem representados. Entende-se desse resultado que as principais funções de apoio ao usuário em termos de suporte e manutenção são implementadas por praticamente todos os softwares, ou seja, em sua maioria apresentam boa preocupação em 
apoiar o seu uso e implantação. Empatados em primeiro lugar estão os DSpace, EPrints e Islandora, com 100\% de itens presentes. Na sequência, o Fedora, com $86 \%$ e o Greenstone com $71 \%$.

\section{Características gerais}

Finalizando a análise das 10 dimensões, apresenta-se, na Figura 10, o resultado da dimensão Características Gerais. Em relação a esta dimensão observa-se que todos estão equiparados, porém, um pouco distantes do valor máximo a ser atingido.

Figura 10 - Funcionalidades das Características Gerais.

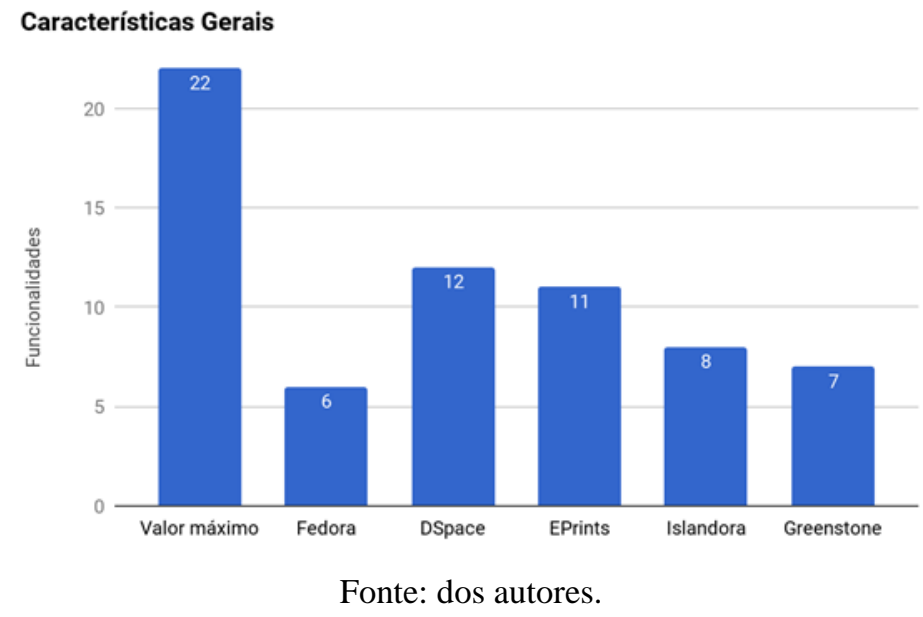

O DSpace alcançou 55\%, seguido do EPrints, com 50\%, logo abaixo, o Islandora, com $36 \%$, o Greenstone, com $32 \%$ e o Fedora, com 27\%. Dá-se destaque a ausência, em todos os repositórios analisados, de aplicação de diretrizes de acessibilidade, tão importantes para promover o acesso por usuários com deficiências temporárias ou permanentes.

Uma das funcionalidades desta dimensão, Grau de usabilidade, não foi avaliada, visto ser necessário um estudo à parte e mais aprofundado.

\section{Total geral das funcionalidades}

Para ilustrar o resultado geral da avaliação, a Figura 11, apresenta os valores obtidos pelos repositórios, considerando todas as dimensões. 
Figura 11 - Funcionalidades de todas as dimensões analíticas.

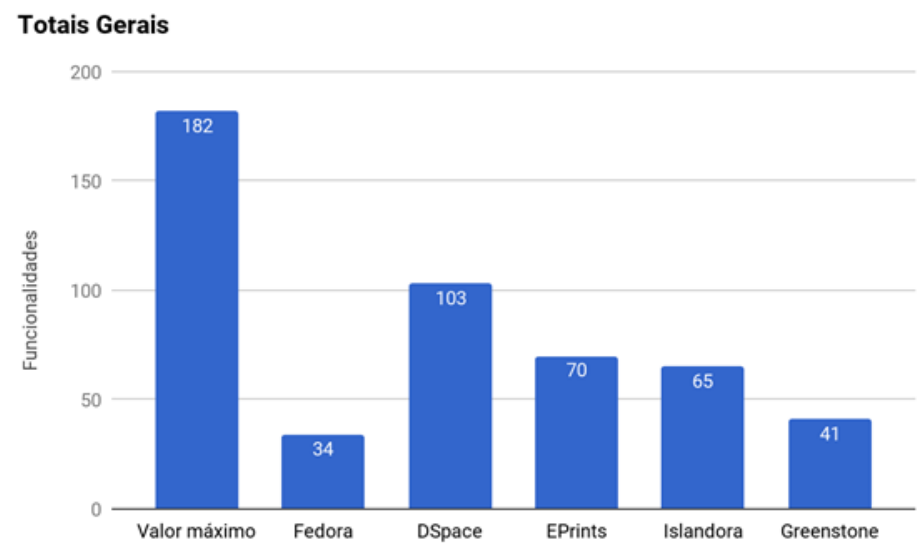

Fonte: dos autores.

Como resultado geral, apresentado na Figura 11, observa-se que o software DSpace se destaca em número de funcionalidades, com $57 \%$, sendo seguido pelo EPrints, com $38 \%$, a Islandora, com 36\%, o Greenstone, com $23 \%$ e o Fedora, com 19\%. Numa avaliação que leve em consideração os aspectos globais da análise, podemos, de fato, inferir que o DSpace é o software livre atualmente mais completo para a gestão de acervos digitais.

No entanto, é importante ressaltar que esse resultado acaba encobrindo potencialidades em determinadas dimensões dos softwares que podem ser mais importantes para um gestor do que outras, o que pode evidentemente variar conforme as demandas de um projeto específico. Logo, entender como os softwares se destacam em cada dimensão é também um resultado importante de sistematizar, o que é apresentado a seguir na Tabela 02.

Tabela 02 - Softwares destaque nas dimensões por número de funcionalidades

\begin{tabular}{ll} 
Dimensão Analítica & Funcionalidades \\
\hline Sistema de Busca & DSpace \\
\hline Sistema de Navegação & DSpace e Islandora \\
\hline Sistema de Organização & EPrints \\
\hline Sistema de Rotulagem e Indexação & DSpace \\
\hline Sistema de Preservação Digital & DSpace \\
\hline Sistema de Interoperabilidade & DSpace \\
\hline Sistema de Administração & DSpace \\
\hline Sistema de Colaboração e Interação Social & DSpace \\
\hline Sistema de Suporte e Manutenção & DSpace, EPrints e Islandora \\
\hline Características Gerais & DSpace
\end{tabular}

Fonte: dos autores

Reafirma-se aqui que o DSpace é destaque majoritário em grande parte das dimensões. 


\section{Conclusão}

Os resultados obtidos nesta pesquisa evidenciam as potencialidades e fragilidades dos principais softwares livres para a gestão de acervos digitais. Foi possível identificar o nível de completude dos softwares e dessa forma, demonstrar que há soluções mais especializadas em determinadas áreas do que em outras.

No entanto, é de suma importância destacar que os resultados foram obtidos não apenas a partir de análise dos softwares instalados, mas principalmente de sua documentação. Assim, percebeu-se que a grande maioria das funcionalidades não são nativas da instalação básica, ou seja, para atingir os objetivos almejados é preciso, além de escolher o software mais adequado, ter uma equipe de profissionais capacitada para instalar e customizar recursos. Além disso, é uma vasta documentação, e nem todas as informações estão disponíveis de forma objetiva.

Como, a princípio, todas as comunidades de desenvolvimento destes softwares livres encontram-se ativas, não é possível projetar um cenário futuro próximo, pois cada software possui potencialidades que podem ser exploradas, transformando-o em uma solução mais completa, podendo ser indicado, sem sombra de dúvidas, para todas as aplicações de gestão de acervos digitais. No entanto, no momento presente, vale ressaltar, o Dspace se consolida como o software mais completo entre os sistemas avaliados.

No entanto, pode ser que outras soluções surjam e tragam inovações importantes para o setor, apresentando avanços significativos na integração, em um único software livre, dessas funcionalidades, em torno das 10 dimensões analíticas avaliadas. Sem dúvida, acarretaria em benefício técnico significativo para o trabalho de gestão de acervos digitais e facilitaria a adoção de padrões universais para a área.

Uma das dimensões que desejamos ressaltar é a de Colaboração e Interação Social que obteve pontuações baixas em todos os repositórios. Com a consolidação do paradigma da Web 2.0 e o uso das redes sociais sendo utilizadas por milhões de usuários, a falta de ferramentas de interação reduz o potencial de experimentação desses softwares e das possibilidades de inclusão dos usuários em novas funções que possam ampliar a participação social no desenvolvimento de novas políticas informacionais. Ainda podemos destacar a “inteligência coletiva" que pode deixar de ser construída a partir das ferramentas de colaboração e interação nesses repositórios. Observa-se, na análise dessa dimensão, um foco em potencial 
que esses softwares poderiam explorar em versões futuras.

Por fim, entende-se que a metodologia utilizada se mostrou eficiente nos resultados que gerou, permitindo produzir as avaliações que apresentamos neste trabalho. Além disso, entende-se também que as dimensões analíticas podem auxiliar projetos de desenvolvimento de tecnologia para a área, servindo como eventuais listas de requisitos, que podem ser analisadas por equipes técnicas para desenvolver funcionalidades específicas em seus sistemas de informação, além de também servirem como listagens para homologação de softwares para editais e pregões públicos para contratação de soluções técnicas.

\section{Referências}

AMBINDER, D. B. M.; MARCONDES, C. H. Novas experiências para apresentação, acesso e leitura de artigos científicos digitais na web. Transinformação, Campinas, v. 25, n. 3 , 2013. Disponível em: 〈http://www.brapci.ufpr.br/brapci/v/a/20456>. Acesso em: 28 mar. 2017.

BANKIER, J. G., GLEASON, K. Institutional repository software comparison. France: United Nations Educational, Scientific and Cultural Organizations, 2014. 16 p.

GOH, D. H.-L. et al. A checklist for evaluating open source digital library. Online Information Review, v. 30, n. 4, p. 360-379, 2006.

KUMAR, K.; RAGHUNADHA, R. T. Analytical study on knowledge about open source software in technological institutional LIS professionals. International Journal of Library and Information Science, v. 5, n. 11, p. 439-446, dec. 2013.

LIHITKAR, S. R.; LIHITKAR, R. S. Open source software for developing digital library: comparative study. Journal of Library \& Information Technology, v. 32, n. 5, p. 393-400, sep. 2012.

MARTINS, D. L.; SILVA, M. F. Critérios de avaliação para sistemas de bibliotecas digitais: uma proposta de novas dimensões analíticas. InCID: Revista de Ciência da Informação e Documentação, Ribeirão Preto, v. 8, n. 1, p. 100-121, abr. 2017. ISSN 2178-2075.

Disponível em: <http://www.revistas.usp.br/incid/article/view/125678/127737> . Acesso em: 12 maio 2017. DOI: 〈http://dx.doi.org/10.11606/issn.2178-2075.v8i1p100-121>.

MINISTÉRIO DA CULTURA. MinC defende criação de uma política nacional de acervos digitais. 2015. Disponível em: http://www.cultura.gov.br/o-dia-a-dia-da-cultura/lasset_publisher/waaE236Oves2/content/minc-defende-criacao-de-uma-politica-nacional-deacervos-digitais/10883. Acesso em: 27 mar. 2017.

MINISTÉRIO DA CULTURA. Plano Nacional de Cultura. 2011. Disponível em: http://www.cultura.gov.br/documents/10883/11294/METAS_PNC_final.pdf/. Acesso em: 27 
mar. 2017.

MINISTÉRIO DA CULTURA. Simpósio sobre acervos digitais. 2010. Disponível em: http://www.cultura.gov.br/o-dia-a-dia-da-cultura//asset_publisher/waaE236Oves2/content/simposio-sobre-acervos-digitais-319218/10877. Acesso em: 27 mar. 2017.

PATEL, D. B. et al. Digital library software DSpace and Greenstone3: a study of features and comparative analysis. E-Library Science Research Journal, v. 2, n. 6, p. 01-07, Apr. 2014.

RANDHAWA, S. Open source software for creation of digital library: a comparative study of Greenstone Digital Library Software \& DSpace. Indian Journal of Library and

Information Science, v. 6, n. 3, p. 45-52, sept./dec. 2012.

PIZZORNO, A. C. P. et al. Buscando soluções para trabalhar o acervo físico, digital e virtual num mesmo ambiente: utilizando o software. Revista ACB: Biblioteconomia em Santa Catarina, Florianópolis, v. 10, n. 1, p. 41-49, 2005. Disponível em:

<http://www.brapci.ufpr.br/brapci/v/a/8095>. Acesso em: 28 mar. 2017.

SUNYE, M. Bibliotecas digitais e os desafios do arquivamento digital, uma visão da ciencia da computação. Encontros Bibli: Revista Eletrônica de Biblioteconomia e Ciência da Informação, Florianópolis, v. 15, n. 29, p. 01-32, 2010. Disponível em: <http://www.brapci.ufpr.br/brapci/v/a/8871>. Acesso em: 28 mar. 2017.

TRAMBOO, S.; HUMMA, S. M. S.; GUL, S. A study on the open source digital library software's: special reference to DSpace, EPrints and Greenstone. International Journal of Computer Applications, v. 59, n. 16, p. 01-09, dec. 2012. 